\title{
Rivoceranib Mesylate
}

National Cancer Institute

\section{Source}

National Cancer Institute. Rivoceranib Mesylate. NCI Thesaurus. Code C74012.

The mesylate salt of rivoceranib, an orally bioavailable, small-molecule receptor tyrosine kinase inhibitor with potential antiang iog enic and antineoplastic activities. Rivoceranib selectively binds to and inhibits vascular endothelial growth factor receptor 2 , which may inhibit VEGF-stimulated endothelial cell migration and proliferation and decrease tumor microvessel density. In addition, this agent mildly inhibits c-Kit and c-SRC tyrosine kinases. 